\section{Hysteresis, Avalanches, and Noise}

Matthew C. Kuntz, Olga Perković, Karin A. Dahmen, Bruce W. Roberts, and James P. Sethna

As computers increase in speed and memory, scientists are inevitably led to simulate more complex systems over larger time and length scales. Although a simple, straightforward algorithm is often the most efficient for small system sizes, especially when the time needed to implement the algorithm is included, the scaling of time and memory with system size becomes crucial for larger simulations.

In our studies of hysteresis and avalanches in a simple model of magnetism (the random-field Ising model at zero temperature), we often have found it necessary to do very large simulations. Previous simulations were limited to relatively small systems (up to $900^{2}$ and $128^{3}$ [宜, see however [3]). In our simulations we have found that larger systems (up to a billion spins) are crucial to extracting accurate values of the universal critical exponents and understanding important qualitative features of the physics.

We have developed two efficient and relatively straightforward algorithms which allow us to simulate these large systems. The first algorithm uses sorted lists and scales as $O(N \log N)$, and asymptotically uses $N \times($ sizeof(double) + sizeof(int)) bytes of memory, where $N$ is the number of spins. The second algorithm, which does not generate the random fields, also scales in time as $O(N \log N)$, but asymptotically needs only one bit of storage per spin, about 96 times less than the first algorithm. Using the latter algorithm, simulations of a billion spins can be run on a workstation with $128 \mathrm{MB}$ of RAM in a few hours.

In this column we discuss algorithms for simulating the zero-temperature random-field Ising model, which is defined by the energy function

$$
\mathcal{H}=-\sum_{<i, j>} J s_{i} s_{j}-\sum_{i}\left[H(t)+h_{i}\right] s_{i},
$$

where the spins $s_{i}= \pm 1$ sit on a $D$-dimensional hypercubic lattice with periodic boundary conditions. The spins interact ferromagnetically with their $z$ nearest neighbors

Matthew C. Kuntz is a Ph.D. candidate at the Laboratory of Atomic and Solid State Physics, Cornell University, Ithaca, NY 14853-2501, mck10@cornell.edu; Olga Perković is with McKinsey \& Company, olga_perkovic@mckinsey.com; Karin Dahmen has just joined the physics faculty of the University of Illinois at Urbana-Champaign; Bruce W. Roberts is working at Starwave Corporation in Seattle, bwr@halcyon.com; James P. Sethna is a professor of physics at Cornell University, sethna@lassp.cornell.edu. Details about his research group can be found at http://www.lassp.cornell.edu/sethna/ with strength $J$, and experience a uniform external field $H(t)$ and a random local field $h_{i}$. We choose units such that $J=1$. The random field $h_{i}$ is distributed according to the Gaussian distribution $\rho(h)$ of width $R$ :

$$
\rho(h)=\frac{1}{\sqrt{2 \pi} R} e^{-h^{2} / 2 R^{2}} .
$$

The external field $H(t)$ is increased arbitrarily slowly from $-\infty$ to $\infty$.

The dynamics of our model includes no thermal fluctuations: each spin flips deterministically when it can gain energy by doing so. That is, it flips when its local field

$$
h_{i}^{\mathrm{eff}}=J \sum_{j} s_{j}+h_{i}+H
$$

changes sign. This change can occur in two ways: a spin can be triggered when one of its neighbors flips (by participating in an avalanche), or a spin can be triggered because of an increase in the external field $H(t)$ (starting a new avalanche).

The zero-temperature random-field Ising model was introduced by Robbins and Ji 33 to study fluid invasion in porous media and front propagation in disordered systems. We have used the same model [5] in a different way 6] to model noise in hysteresis loops in disordered materials. In particular, we wish to understand Barkhausen noise in magnetic materials with quenched disorder 伍. It has been found experimentally that when an external field is gradually applied, many materials magnetize not continuously, but in a noisy way, with jumps (avalanches) of all sizes. (The noise can be heard by wrapping the magnetizing material in a coil of wire and amplifying the signal into a speaker. The signal makes a crackling noise when a permanent magnet is brought close, quite similar to the crackling noises heard in fires, crisped rice cereals, and crumpled paper [7].) In the steepest part of the hysteresis loop, these avalanches are found to have a power-law distribution of sizes with an exponent $\tau \approx 1.5$. Power laws are also found in the distribution of avalanche times with an exponent $\alpha \approx 2$ and in the power spectrum.

The zero-temperature random-field Ising model is interesting because, as in the disordered magnetic materials it attempts to model, the avalanches can have a broad range of sizes. If all the avalanches were small, understanding them would be straightforward and not very interesting. Indeed, at large disorder $R$, the chance that a spin which has just flipped will trigger one of its $z$ neighbors scales roughly as $z J / R$. If this quantity is smaller than unity (large disorder), all avalanches will be small: the noise will be a series of small pops all of about the same size. This behavior is uninteresting not because it is simple, but because the behavior is strongly depen- 
dent on the details of the model at short distances, where the model is at best a caricature of a real material.

It also is easy to understand the system in the small disorder regime $z J / R>>1$, where almost all the spins flip over in one infinite avalanche. There are many problems (for example, fracture and first-order phase transitions) where a single nucleation event leads to the release of the stored energy in a single catastrophic event.

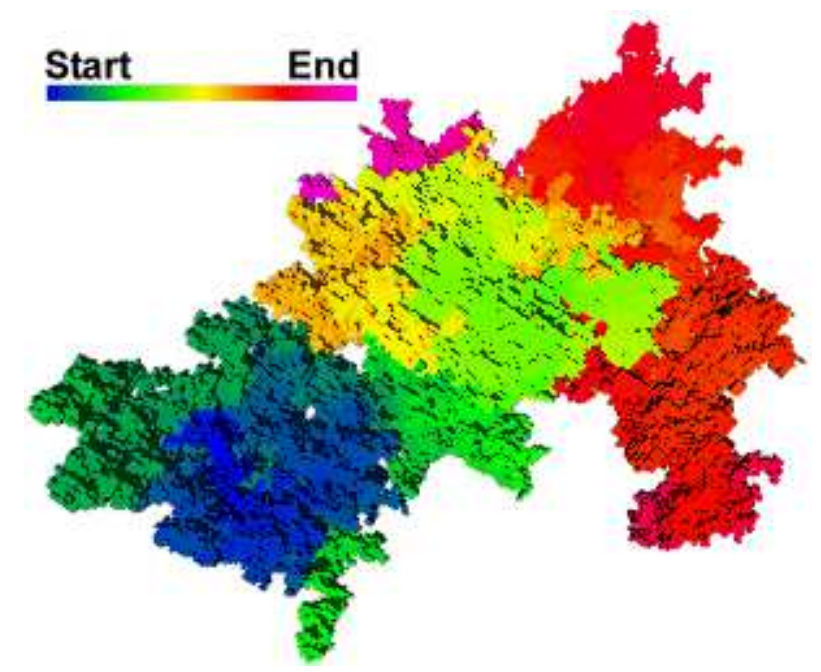

FIG. 1. A three-dimensional view from one side of a single avalanche in a $200 \times 200 \times 200$ system at $R=2.3$ (within $6 \%$ of the critical disorder $R_{c}$ ). The avalanche contains 282,785 spins. The time when each spin flipped is shown by its color. The avalanche generally grew from left to right. Note that it has many branches and holes; the large avalanches in three dimensions probably have a fractal dimension a little less than three. Also notice that on the right hand side, there are several dark red spots poking through in the middle of the light green area. The green area stopped growing, but other parts of the avalanche later filled in the holes.

We focus on the crossover between these two limiting cases, where the system exhibits crackling noise with avalanches of all sizes. For a particular value of the disorder $R=R_{c}$, a spin which has just flipped will on average flip exactly one neighbor as the external field $H(t)$ is increased to a particular value $H_{c}$. The avalanches at $R_{c}$, $H_{c}$ (the critical point), are finely balanced between stopping and growing forever. They advance in fits and starts (see Figs. 1 and 2) and come in all sizes (Figs. 3 and 4) with a probability which decreases as a power law of the number of spins in the avalanche. At $H_{c}$, the distribution of avalanche sizes decays with an exponent of $\tau \approx 1.6$ (quite close to the experimental results), and integrated over all $H$, the distribution decays with an exponent 13] $\tilde{\tau} \approx 2$. Below the critical disorder $R_{c}$, there will be an avalanche which will flip a nonzero fraction of the spins in the system even as the system size goes to infinity: we call this avalanche the infinite avalanche. There are very large avalanches even for disorders far above the critical disorder. In three dimensions, there are still two decades of power law scaling $50 \%$ above the critical point. However, the convergence to the expected asymptotic power law is very slow (Figure 3). This behavior means that we see critical scaling even if we do not fine tune $R$ to $R_{c}$, but we must use very large systems to get close enough to $R_{c}$ to obtain a convincing power law. In practice, we needed simulations of approximately a billion spins to understand the physics in three dimensions [10]. Two dimensions remains a challenge because the proper scaling is not clear even for $30,000^{2}$ spins 10,14 .

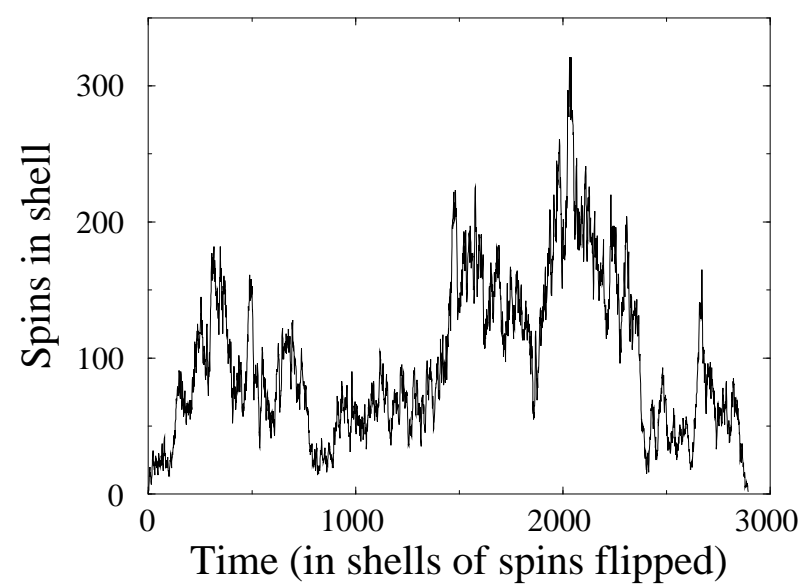

FIG. 2. A time series showing the number of spins which flipped in each shell of the avalanche shown in Fig. 19 [9]. Note that the avalanche is a series of bursts: near the critical point, the avalanche is always on the verge of halting, so it proceeds in fits and starts.

It is crucial with this many spins that our algorithms be efficient both in computer time and memory. We begin by giving the simple, but inefficient approach which has an execution time which scales as $O\left(N^{2}\right)$. We then develop a more efficient approach using a sorted list which gives an execution time which scales as $O(N \log N)$, but which needs memory storage which scales as $N \times(\operatorname{sizeof}($ double $)+\operatorname{sizeof}($ int $))$. A billion spins would demand 12 Gigabytes of RAM for efficient execution, which is not usually available. Finally, we give an algorithm whose execution time also scales as $O(N \log N)$, but whose memory requirements are asymptotically only one bit per spin. In this case $10^{9}$ spins requires $120 \mathrm{MB}$ of storage, which is feasible on a standard workstation [8]. We conclude with a discussion of time and space issues for calculating and storing histograms and correlation functions. 


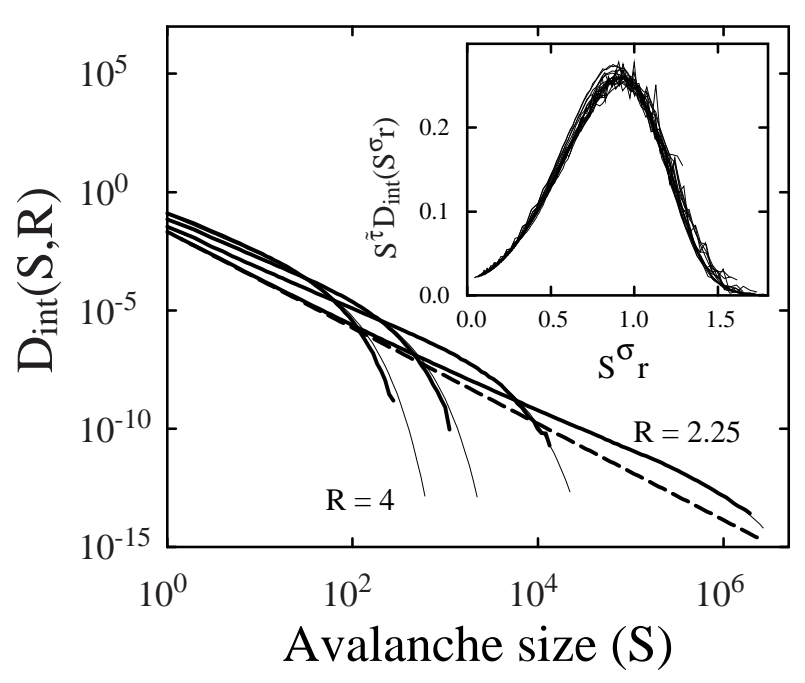

FIG. 3. Distribution of avalanche sizes for different values of the disorder $R$ in three dimensions. Some avalanches remain large (hundreds of spins) for $R$ a factor of two above the critical value $R_{c} \sim 2.16$ where we expect a pure power law. The avalanches are enormous (millions of spins) when the system is still $4 \%$ away from the critical point; for this reason we need large systems. The inset is a scaling collapse of the data: the thin lines in the main figure show the scaling prediction for the avalanche sizes stemming from the scaling collapse 10. Note that the scaling predictions already work well at $R=4$. The pure asymptotic power law behavior is not yet seen at $R=2.25$, when six decades of scaling are observed. We needed simulations of a billion spins to show convincingly that the power law would eventually occur [10].

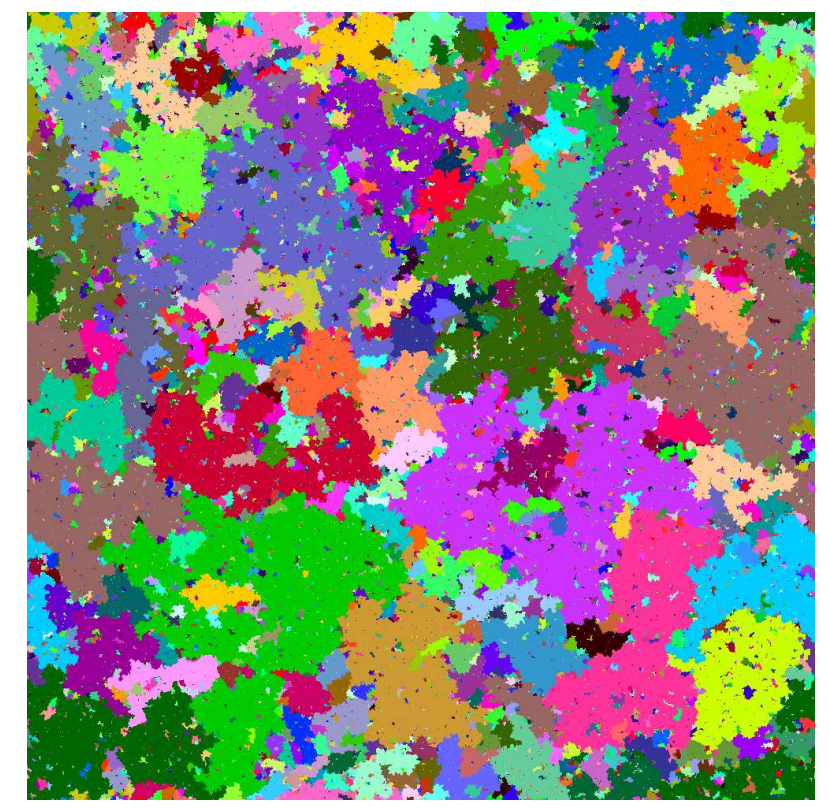

FIG. 4. A 30,000 × 30,000 simulation with disorder $R=0.65$, where each pixel represents a $30 \times 30$ square, and each avalanche is a different color. Note that there are avalanches of all sizes, with many smaller avalanches, and fewer large ones.

\section{The Brute Force Method}

The brute force method is the easiest one to implement and is competitive for system sizes up to about 10,000 spins. In this method, we store a spin direction and a random field for each site of the lattice. We can then proceed as an experimentalist would by measuring the magnetization at specific predetermined values of $H$. We start with magnetization $M=-N$ and a large negative field $H_{0}$ and then increment to $H_{1}$, check all spins in the lattice, and flip those spins in a positive local field. Then we must check the neighbors of the flipped spins again to see if their local fields are now positive. This procedure is continued until all the neighbors of flipped spins have been checked. We then repeat the whole procedure again for a new field $H_{2}$, and so on. This approach gives the correct magnetization at the fields $H_{n}$ : the order in which spins are flipped can be shown not to influence the final state [15,5]. However, unless the increments in $H$ are very small, several avalanches may occur in a given increment, and all information about single avalanches (such as histograms of avalanche sizes) will be distorted.

The time for the brute force method scales as $O(N X T)$, where $X$ is the number of fields $H_{n}$ at which the magnetization is measured, and $T$ is the average time needed to check the neighbors of the flipped spins measured in units of shells of neighbors.

Lattice
\begin{tabular}{|r|r|r|r|r|}
\hline 1 & 2 & 3 & 4 & 5 \\
\hline 6 & 7 & 8 & 9 & 10 \\
\hline 11 & 12 & 13 & 14 & 15 \\
\hline 16 & 17 & 18 & 19 & 20 \\
\hline 21 & 22 & 23 & 24 & 25 \\
\hline
\end{tabular}

\begin{tabular}{|c|c|c|c|c|c|c|c|}
\hline 11 & 17 & 9 & 10 & 20 & 20 & 18 & 6 \\
\hline 12 & 12 & 15 & 15 & 19 & 19 & 7 \\
& & & & & &
\end{tabular}

End of Shell

FIG. 5. Example of how a queue is used to propagate an avalanche. The colored spins are spins which either have flipped in the current avalanche or will flip in the current avalanche. Spin 13 triggered the avalanche, then the light grey spins $(14,8,12)$ were put on the queue as the first shell. As they flipped, the second shell, the blue spins $(15,19,7,11,17)$, were put on the queue. As the first blue spins $(15,19,7)$ flipped, the dark red spins $(10,20,20,18,6)$ were added to the queue as the start of the third shell. The next spin to flip is at the left hand side of the queue. When this spin flips, its neighbors will be checked, and the neighbors which are ready to flip will be added to the right hand side of the queue. The small numbers below the spins in the queue indicate which neighbor caused the spin to be put on the queue. Note that different neighbors can cause a spin (such as spin 20) to be put on the queue more than once. We have to be careful to only flip the spin once.

A variation on this approach is to propagate one avalanche at a time as shown in Fig. 5: 
1. Find the triggering spin for the next avalanche by checking through the lattice for the unflipped site with the largest internal field $h_{i}^{\text {int }}=h_{i}^{\text {eff }}-H$.

2. Increment the external field so it is just large enough to flip the site, and push the spin onto a first-in first-out (FIFO) queue (see Fig. 5, right).

3. Pop the top spin off the queue.

4. If the spin has not been flipped, flip it and push all unflipped neighbors with positive local fields onto the queue.

5. While there are spins on the queue, repeat from step 3 .

6. Repeat from step 1 until all spins are flipped.

This method is standard for avalanche propagation problems. It also is related to the propagation of cluster flips in the Wolff algorithm [16]. Using a queue instead of recursion has two advantages. First, recursion is slower and more memory intensive, because each recursive call must push all local variables and all registers onto the system stack (which usually has a pre-allocated limit). If we use our own queue, we need only to push the coordinate of the next spin on the queue each time, and we can make the queue as large as necessary. Second, in order to produce a natural spin-flip order, we want to flip all spins that are ready to flip at a given time before we flip the spins that they cause to flip. If we put spins that are ready to flip on a FIFO queue, we correctly achieve this order. This procedure corresponds to doing a breadthfirst search. Recursion, which is the same as putting the spins on a LIFO stack, would explore all possible consequences of flipping the first neighbor it looks at before it considers the second neighbor. This depth-first search produces an unnatural spin-flip order (although the final state after the avalanche is unchanged [15.5). The dynamics during the avalanche of Fig. 2 assumes one shell of spins flipped during each time slice, which is easy to determine by placing markers on a FIFO spin queue, as shown in Fig. 5. Each time the marker is popped off of the queue, a new shell is started and the marker is put back on the end of the queue.

Doing the brute force algorithm one avalanche at a time is very inefficient except at very low disorders. Sweeping through the entire lattice for each avalanche takes $O(N)$ time per avalanche. Because there are $O(N)$ avalanches, the total running time scales as $O\left(N^{2}\right)$. A hybrid approach, finite steps in field followed by internal propagation of avalanches, could be quite efficient if one is solely interested in the magnetization at those fields. A brute force method is probably necessary when simulating systems with long-range interactions [2].

\section{Time Efficiency: Sorted Lists}

The brute force method is very inefficient at locating the origin of the next avalanche, and we are immediately led to think of storing the several largest local fields in each sweep. If we take this thinking to its logical conclusion, we are led to store a list of all of the spins in the system, sorted according to their random fields.

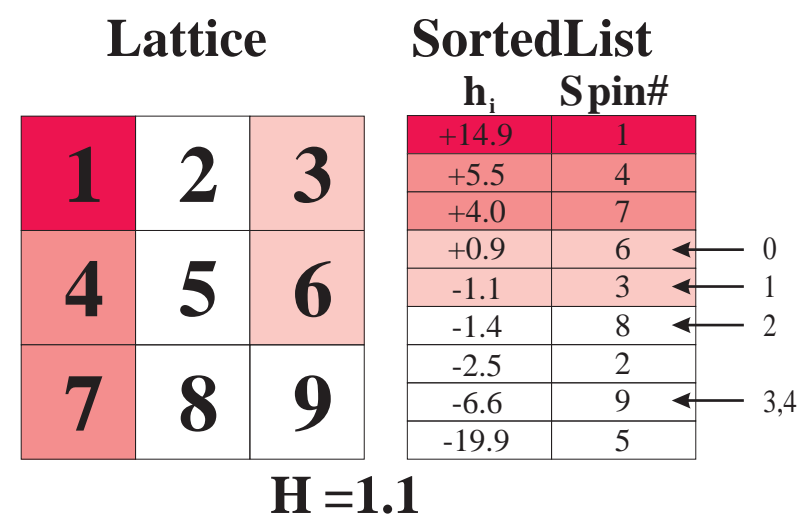

FIG. 6. Example of how a sorted list is used to find the next spin in the avalanche. The colors indicate spins which have already flipped. The first column in the sorted list contains the random field, and the second column contains the number of the spin with that random field. The arrows to the right indicate the nextPossible $\left[n_{\uparrow}\right]$ pointers — the first spin which would not flip with $n_{\uparrow}$ neighbors up. The spins pointed to are the possible starting locations for the next avalanche. Note that some of the pointers point to spins that have already flipped, meaning that these spins have more than $n_{\uparrow}$ neighbors up. In a larger system, the unflipped spins will not all be contiguous in the list.

Unfortunately, life is complicated by the fact that spins experience not only their local random fields, but also fields from their neighbors. To find the origin of the next avalanche, we use the following algorithm:

1. Define an array nextPossible $\left[n_{\uparrow}\right], n_{\uparrow}=0,1 \ldots z$, which points to the location in the sorted list of the next spin which would flip if it had $n_{\uparrow}$ neighbors. Initially, all the elements of nextPossible $\left[n_{\uparrow}\right]$ point to the spin with the largest local random field, $h_{i}$.

2. Choose from the $z+1$ spins pointed to by nextPossible, the one with the largest internal field $h_{i}^{\mathrm{int}}\left[n_{\uparrow}\right]=n_{\uparrow}-n_{\downarrow}+h_{i}=2 n_{\uparrow}-z+h_{i}$.

3. Move the pointer nextPossible $\left[n_{\uparrow}\right]$ to the next spin on the sorted list.

4. If the spin with the largest $h_{i}^{\text {int }}\left[n_{\uparrow}\right]$ has $n_{\uparrow}$ up neighbors, then flip it. Otherwise go back to step 2 .

An example of the sorted list and the pointers from nextPossible is shown in Fig. 6 . 
The sorting of spins can be done in time $O(N \log N)$. Storage with this algorithm is $N \times$ sizeof(int) for the sorted array (if we reduce the $D$ dimensional coordinates to one number [18), and $N \times$ $($ sizeof(spin) $+\operatorname{sizeof}($ double $))$ for the lattice itself. Various other compromises between speed of execution and storage are possible, but all leave the running time $O(N \log N)$. The sorted-list algorithm is fast: the largest system sizes we can store on a reasonable workstation execute $1000^{2}$ and $100^{3}$ spins in a few seconds. It is the method of choice for these small systems or when one is interested in the behavior for non-monotonically increasing fields. 用

\section{Space Efficiency: One Bit per Spin}

The combination of the rapid execution of the sorted-list algorithm and large finite size effects led us to develop an algorithm optimized for memory efficiency. The key is to recognize that we need never generate the random fields! In invasion percolation 19] (and in the interface problem [3] analogous to ours) the random fields are generated only on sites along the boundary of the growing cluster. In our problem, we can take this idea further: for each change in a spin's local field given by Equation 3, we generate only the probability that it will flip. Storing the random fields is unnecessary because the external field, the configuration of the spin's neighbors, and the knowledge that the spin has not yet flipped gives us all the information which we need to determine the probability that the spin will flip. The only quantity which we must store for each site of the lattice is whether the spin is up or down. Thus, we can store each site of the lattice as a computer bit saving large amounts of memory.

For a monotonically increasing external field, the conditional probability that a spin flips before its nonrandom local field, $H_{\mathrm{nr}} \equiv H+\left(2 n_{\uparrow}-z\right)$, reaches $H_{\mathrm{nr}}+\Delta H_{\mathrm{nr}}$ given that it has not flipped by $H_{\mathrm{nr}}$ is

$$
P_{\text {flip }}\left(H_{\mathrm{nr}}, \Delta H_{\mathrm{nr}}\right)=\frac{\left[P_{\downarrow}\left(H_{\mathrm{nr}}\right)-P_{\downarrow}\left(H_{\mathrm{nr}}+\Delta H_{\mathrm{nr}}\right)\right]}{P_{\downarrow}\left(H_{\mathrm{nr}}\right)},
$$

where $P_{\downarrow}\left(H_{\mathrm{nr}}\right)$ is the probability that a spin points down

\footnotetext{
${ }^{*}$ The sorted-list algorithm can be used for non-monotonically increasing fields with only a few minor additions. When the external field is being lowered instead of raised, the avalanche propagation is the same, except spins are flipped when their local field becomes less than zero instead of when it becomes greater. The nextPossible array needs to be handled carefully. The next spin that would flip up if the field were increased is the last spin that would have already flipped down with the field decreasing. Every time the direction of change of the external field is reversed, all of the nextPossible $\left[n_{\uparrow}\right]$ pointers need to be adjusted by one to account for this.
}

when the local field is $H_{\mathrm{nr}}$. A spin with local field $H_{\mathrm{nr}}$ will point down if its random field $h_{i}$ satisfies $h_{i}+H_{\mathrm{nr}} \leq 0$. This condition implies that the probability that a spin with $n_{\uparrow}$ up neighbors points down is

$$
\begin{aligned}
P_{\downarrow}\left(n_{\uparrow}, H\right) & =\int_{-\infty}^{-H_{\mathrm{nr}}\left(n_{\uparrow}, H\right)} \rho(h) d h \\
& =\frac{1}{2}+\frac{1}{2} \operatorname{erf}\left(-H_{\mathrm{nr}}\left(n_{\uparrow}, H\right) / \sqrt{2} R\right) \\
& =\frac{1}{2} \operatorname{erfc}\left(H_{\mathrm{nr}}\left(n_{\uparrow}, H\right) / \sqrt{2} R\right) .
\end{aligned}
$$

(Writing $P_{\downarrow}$ in terms of the erfc function removed some problems with rounding at large negative fields $H$.) These probabilities are illustrated graphically in Fig. 目.

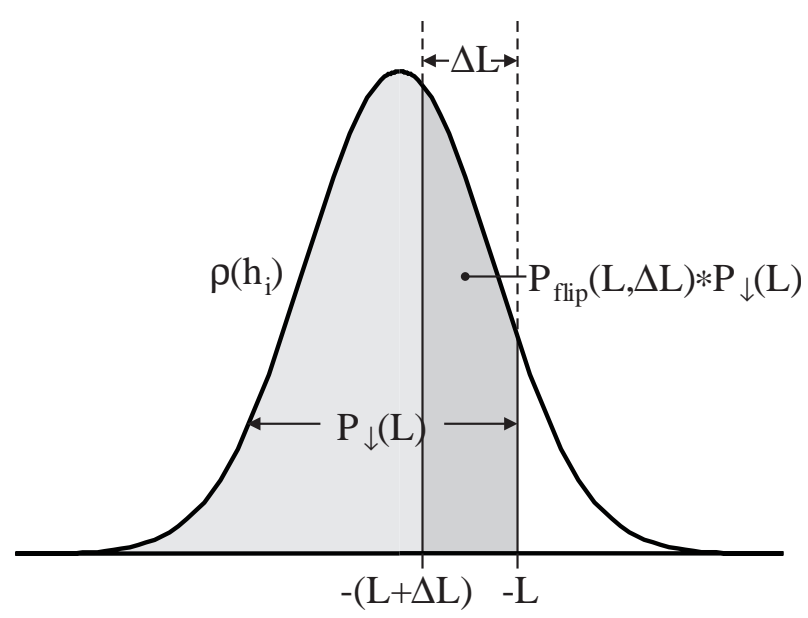

FIG. 7. The probability that a spin will not have flipped by the time its local field reaches $L$ is the probability that the random field is less than $-L$. This probability is represented by the shaded area of the Gaussian. The probability that the spin will flip before the field reaches $-(L+\Delta L)$ is represented by the area of the darker region divided by the area of the shaded region.

Finding the next avalanche is subtle when the random fields are not stored: changing the external field $H$ introduces a probability that any unflipped spin in the lattice may flip. Inspired by the continuous time MonteCarlo algorithm [20], we keep track of $N_{n_{\uparrow}}$, the number of down spins which have $n_{\uparrow}$ up neighbors. Given the probabilities that spins with $n_{\uparrow}$ up neighbors will flip, we calculate both the change in the external field $\Delta H$ needed to flip the next spin and the probability that the next spin to flip has $n_{\uparrow}$ up neighbors. We then randomly choose $n_{\uparrow}$ and search at random through the lattice for a spin with $n_{\uparrow}$ up neighbors. The time taken for the search is the part of the algorithm which scales worst for large $N$. If there are $N_{n_{\uparrow}}$ spins left, this search will take an average time $O\left(N / N_{n_{\uparrow}}\right)$. Summing over $N_{n_{\uparrow}}$ and $n_{\uparrow}$ yields a bound of order $z N \log N$. In one of our programs, we use a tree structure to do this search more efficiently; 
this complication decreases the running time by $40 \%$ for a $500^{2}$ system at $R=1$.

How do we calculate $\Delta H$ ? From Equation 1 , the probability that a single spin with $n_{\uparrow}$ neighbors up has not flipped in the range $\Delta H$ is $1-P_{\text {flip }}\left(n_{\uparrow}, H, \Delta H\right)=$ $P_{\downarrow}\left(n_{\uparrow}, H+\Delta H\right) / P_{\downarrow}\left(n_{\uparrow}, H\right)$. The probability that no spin with $n_{\uparrow}$ up neighbors has flipped in this range is

$$
P_{n_{\uparrow}}^{\text {none }}=\left[\frac{P_{\downarrow}\left(n_{\uparrow}, H+\Delta H\right)}{P_{\downarrow}\left(n_{\uparrow}, H\right)}\right]^{N_{n_{\uparrow}}},
$$

and the probability that no spin has flipped between $H$ and $H+\Delta H$ is

$$
P^{\text {none }}(\Delta H)=\prod_{n_{\uparrow}=0}^{z} P_{n_{\uparrow}}^{\text {none }} .
$$

To find $\Delta H$, we choose a random number $r$ uniformly distributed between zero and one, and set $\Delta H$ so that $P^{\text {none }}(\Delta H)=r$.

Unfortunately, we cannot solve for $\Delta H$ analytically, and we must find a numerical solution. To find this solution efficiently, we need a good initial guess. In analogy with nuclear decay, if spins flip with a constant rate $\Gamma$, we expect the probability that no spins have yet flipped to be $e^{-\Gamma \Delta H}$. So, for a first approximation of $\Delta H$, we assume that the spin-flip rate $\Gamma$ is a constant, and therefore

$$
\Delta H_{1}=-\frac{\log (r)}{\Gamma(H)}
$$

where $\Gamma(H)$ is given by

$$
\begin{aligned}
\Gamma(H) & =-\frac{d \log \left(P^{\text {none }}(\Delta H=0)\right)}{d \Delta H} \\
& =-\sum_{n_{\uparrow}=0}^{z} N_{n_{\uparrow}} \frac{d \log \left(P_{\downarrow}\left(n_{\uparrow}, H+\Delta H\right)\right)}{d \Delta H} \\
& =\sum_{n_{\uparrow}=0}^{z} N_{n_{\uparrow}} \frac{\rho\left(H_{\mathrm{nr}}\left(n_{\uparrow}, H\right)\right)}{P_{\downarrow}\left(n_{\uparrow}, H\right)} \equiv \sum_{n_{\uparrow}=0}^{z} \Gamma\left(n_{\uparrow}, H\right)
\end{aligned}
$$

We can make a better second guess by looking at the error in our first guess. If the error in our guess is $\Delta r=$ $P^{\text {none }}(\Delta H)-r$, then we can make an improved second guess for $\Delta H$ by aiming for $r-\Delta r$ :

$$
\Delta H_{2}=-\frac{\log (r-\Delta r)}{\Gamma(H)} .
$$

These two guesses can then be used as input into a root finding routine [21]. Note that while these guesses are usually very good for small $|H|$ and lead to quick solutions, they can be very bad for large $|H|$. If the guesses for $\Delta H$ are very large, it may be better to choose two arbitrary guesses. In our code, if $\Delta H_{1}>20$, we use $\Delta H_{1}=0$ and $\Delta H_{2}=20$ for the two guesses.

Our algorithm for finding the next avalanche becomes

1. Choose a random number $r$ uniformly distributed between zero and one.

2. Pre-calculate the values of $P_{\downarrow}\left(n_{\uparrow}, H\right)$ using Equation 6 . These values will be used repeatedly in solving for $\Delta H$.

3. Calculate guesses for $\Delta H$ using Equations 9 and 11, and use them as input to a root finding routine to find the exact solution for $\Delta H$.

4. Increment $H$ by $\Delta H$.

5. Calculate the array probFlip $\left[n_{\uparrow}\right]$ for use in the remainder of the avalanche, where probFlip $\left[n_{\uparrow}\right]$ is the probability at the current field $H$ that a spin will flip when its number of up neighbors changes from $n_{\uparrow}$ to $n_{\uparrow}+1$ (see Equation ().

6. Calculate the rates for flipping spins for each $n_{\uparrow}$ at the current field $H=H_{\mathrm{old}}+\Delta H$ :

$$
\Gamma\left(n_{\uparrow}, H\right)=N_{n_{\uparrow}} \rho\left(H_{\mathrm{nr}}\left(n_{\uparrow}, H\right)\right) / P_{\downarrow}\left(n_{\uparrow}, H\right)
$$

and the total rate $\Gamma(H)=\sum_{n_{\uparrow}=0}^{z} \Gamma\left(n_{\uparrow}, H\right)$.

7. Choose a random number uniformly distributed between zero and $\Gamma$ and use it to select $n_{\uparrow}$.

8. Search at random in the lattice for an unflipped spin with $n_{\uparrow}$ up neighbors 22].

9. Start the avalanche at that spin. During an avalanche, the algorithm is essentially the same as the brute force algorithm:

10. Push the first spin onto the queue.

11. Pop the top spin off of the queue.

12. If the spin is unflipped, flip it, find $n_{\uparrow}$, and decrease $N_{n_{\uparrow}}$ by one. Otherwise, skip to step 14.

13. Look at all unflipped neighbors. For each unflipped neighbor, find the current number of up neighbors, $n_{\uparrow}$; decrease $N_{n_{\uparrow}-1}$ by one, and increase $N_{n_{\uparrow}}$ by one. Push the spin on the queue [23] with probability probFlip $\left[n_{\uparrow}-1\right]$, as calculated in step (5).

14. While there are spins left in the queue, repeat from step 11.

15. While there are unflipped spins, repeat from step 1. 
This algorithm is about half as fast in practice as the sorted-list algorithm which is faster than we expected. The overhead involved in solving for $\Delta H$ is presumably compensated by the time saved not shifting data in and out of cache. Systems of $10^{9}$ spins take a few days of CPU time on a reasonable workstation; $30,000^{2}$ systems take less than 15 hours on a $266 \mathrm{MHz}$ Pentium II.

\section{Calculating Histograms and Correlations}

Several functions are needed to characterize the critical properties of our model. The simplest function is the magnetization $M$ as a function of the external field $H$. We also calculate distributions of avalanche sizes (Fig. 3, top), and correlation functions. Some care must be taken to make sure that the calculation of these functions does not dramatically increase the running time or memory requirements of the simulation.

When doing calculations with a billion spins, we cannot output any quantity which scales linearly with the system size. Instead of computing $H(M)$ at each avalanche (about a GB of data, which rapidly would fill our disk), we are forced to compute $H\left(M_{n}\right)$ at pre-chosen points.

The characteristic feature of the critical point is the appearance of an infinite avalanche. The equivalent of an infinite avalanche in a finite system is an avalanche which spans the entire system in at least one dimension. To tell whether we are above or below the critical point, we need to detect these spanning avalanches. In three and higher dimensions, the number of spanning avalanches as a function of $R$ is also interesting to study. The most obvious way of detecting spanning avalanches is to mark each row as a spin flips in it, and check at the end of the avalanche to see if all the rows contain flipped spins from the avalanche. However, this method requires $\mathrm{O}\left(N^{1 / D}\right)$ operations per avalanche. Because there are many small avalanches, this method is unacceptable. A preferable method is to keep track of the $2 \times D$ boundaries of the avalanche as it grows. If a pair of boundaries meet, then the avalanche is a spanning avalanche. We must take care to treat the periodic boundary conditions properly.

Another useful function is the avalanche size distribution $D(S)$, defined as the number of avalanches which flip $S$ spins during the simulation, divided by the total number of spins. Like the $M(H)$ curve, the avalanche size distribution scales linearly with the system size. Thus, we need bins up to size $N$, the size of the largest possible avalanche. Logarithmic binning is the obvious solution, with bin $n$ including all sizes $b_{a}^{n-1}<S<b_{a}^{n}$. We have chosen $b_{a}$ from 1.01 to 1.1. Large bins are preferable for lower statistical noise. This choice is particularly important in the tail of very large avalanches, where small bins would contain few avalanches. However, very large bins will systematically alter the shape of the scaling functions (although they will not change the critical exponents). It is important to divide the final population in each bin by the number of integers contained within the bin (and not just the bin width). Clearly we should also ignore the early bins which do not contain any integers.

We calculate the correlation function $G(x, R)$ within an avalanche, where $G(x, R)$ gives the probability that the first spin in an avalanche will cause a spin a distance $x$ away to flip in the same avalanche. At the beginning of each avalanche, we record the coordinates of the first spin in the avalanche. Then, for each subsequent spin in the avalanche, we calculate the distance $x$ to the first spin, and add one to the appropriate bin. Logarithmic binning is not necessary for the correlation function, because the size of the correlation function is proportional to the length of the system, not the total number of spins. Thus, we use a fixed bin size $b_{c}=1$. At the end of the simulation, each bin should be normalized by the number of spins which are between $x-b_{c} / 2$ and $x+b_{c} / 2$ away from the origin.

The only tricky part of calculating $G(x, R)$ comes from the periodic boundary conditions. If the avalanche crosses a boundary, two points at opposite ends of the avalanche can come close together. Because we do not calculate $G(x, R)$ for spanning avalanches, we know that there will be at least one row in every dimension which is not touched by the avalanche. To calculate separations, we use the periodicity of the lattice and the continuity of the avalanche to shift the coordinates so they are all on one side of these empty rows. Because we are already keeping track of the boundaries of the avalanche for the detection of spanning avalanches, finding an empty row is easy.

The running times of the three algorithms as a function of system size are shown in Fig. 8. The brute force algorithm can be useful when one cares only about $M(H)$ at a few points, but is otherwise too slow for large systems. The sorted-list algorithm is the fastest algorithm, but on a $128 \mathrm{MB}$ machine, only system sizes of about six million spins can be run. The bits algorithm is almost as fast as the sorted-list algorithm, and asymptotically uses only one bit of memory per spin. 


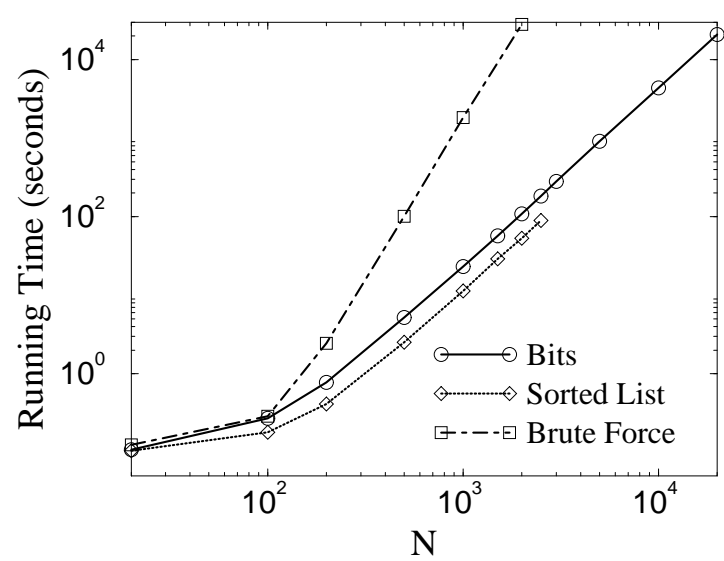

FIG. 8. The running times for the three algorithms for two-dimensional systems with $R=1.0$ on a $266 \mathrm{MHz}$ Pentium II with $128 \mathrm{MB}$ of memory. Note that both the bits algorithm and the sorted-list algorithm have run times which grow approximately linearly (the $\log N$ is not visible), and the brute force running time grows quadratically. Also notice that the largest bits simulation was 64 times larger than the largest sorted-list simulation.

We have made $\mathrm{C}++$ source code implementing all three algorithms available at http://www.lassp.cornell.edu/sethna/hysteresis/code/. Each algorithm is contained in a separate, welldocumented class. There are classes for detecting spanning avalanches, measuring avalanche size distributions, and measuring correlation functions. There are also both Microsoft Windows and command line interfaces to the code. The command line interface should be portable to any computer with a $\mathrm{C}++$ compiler and an implementation of the Standard Template Library. We have also compiled executables for Windows 95/NT, Linux, and several other flavors of UNIX. The running times in Fig. 8 all come from the code compiled under Linux.

Working on hysteresis, avalanches and noise with our model has been very rewarding. The simulations are beautiful and entertaining in themselves. Developing faster and more space efficient algorithms was amazingly satisfying: each new method not only eased our lives and our computer budgets, but also opened a whole new window on the behavior of the model. It was also fun developing new ways of measuring what was happening in the simulations: watching the spins flip and measuring the avalanche size distribution was only the beginning. We have written a few exercises which we hope will entertain and inform you as they did us.

\section{Suggested problems for further study}

1. Phase Transitions in the Shape of the Loop. Download our program from http://www.lassp.cornell.edu/sethna/hysteresis/code/.
Also download DynamicLattice and xmgr if you are using Unix. Run the program. Under Windows, just press OK in the opening dialog box to run a simulation with the default parameters. Under UNIX, type run at the $\gg>$ prompt. (More detailed instructions can be found on our Web site.) Try the other two algorithms. For smaller systems, brute force works acceptably, but for $L=500$ it is rather slow.

You should see an animation of the avalanches as the external field is ramped upward and the spins flip. After the simulation ends, you should obtain a graph of $M(H)$, the avalanche size distribution $D(S, R)$, and the correlation function $G(x, R)$. The $M(H)$ curve shows the bottom half of the hysteresis loop: it should consist of many jumps of various sizes. The top half of the hysteresis loop is pretty much the same shape $-M(-H)$, but the details of the jumps are different.

The avalanche size distribution $D(S)$ measures the number of jumps as a function of size $S$. It should look like a fairly good power law, and be a straight line on a $\log -\log$ plot.

A log-log plot of the correlation function $G(x, r)$ looks much less linear. At small distances, the correlation function decreases as a power law, but the power law behavior bends over after only a decade or so. This behavior is a symptom of $R$ not being quite at the critical disorder $R_{c}$.

(a) According to our scaling theory [5, 10], near the critical point $D\left(S, R_{c}\right) \sim S^{-\tilde{\tau}}$. What is your best estimate for the exponent $\tilde{\tau}$ at the default value $R=1.0$ ?

(b) Do a simulation at a smaller value of disorder $R$, say 0.8. Does the behavior of $G(x, R)$ remain a power law over larger values of $x$ ?

(c) Do a simulation with $D=3, L=50$, and $R=2.5$. What is $\tilde{\tau}$ in three dimensions? (To obtain better data, do several runs and use the averaging option.) Consider $R=2.1$. Does the shape of the $M(H)$ curve look qualitatively different? We believe that the power law distributions occur for $R_{c} \sim 2.16$. At this value, the hysteresis loop first develops a macroscopic jump (the infinite avalanche). One obtains power laws and scaling at the phase transition in the shape of the hysteresis loop, between smooth loops and ones with an infinite avalanche.

2. Time, Space, and Bits. We have a local, somewhat older supercomputer with four gigabytes of RAM. How large a system could we run in this memory using the three algorithms we discussed? Ignore all the memory requirements except those that scale linearly with the number of spins $N$; the rest is negligible. Assuming the time spent in bits continues to grow as $N \log N$ after the last data point in Fig. 8, how long will the largest possible bits simulation take to complete? 
3. Programming. To do the following problems, you will need to download the source code for our program from http://www.lassp.cornell.edu/sethna/hysteresis/code/.

You will also need a $\mathrm{C}++$ compiler which supports the standard template libraries. The standard template libraries are an important advance for scientific programming. We find them incredibly useful, and look forward to their wide implementation now that the $\mathrm{C}++$ standard is in place. Links to compilers supporting the standard template libraries can be found on our Web page.

Run the simulation described in Problem 1 to test that the program works. Then try the following problems (many details on how to work with our source code can be found on our Web site).

(a) Adding a Spin-Flip Action: Time Series for Large Avalanches. We have designed our code so that it is easy to add new types of measurements. One quantity which experimentalists measure is the change in magnetization with time. In our code, we record the time series of the whole run, with each avalanche represented as a single point. There is also interesting structure within each avalanche (see Fig. 2). Add a new class to the program which records the time series within the largest avalanche.

(b) Implementing Brute Force. Implement the brute force algorithm. You can either replace the BruteForceHysteresisSimulation class or implement it from scratch.

(c)

Implementing

Sorted

Lists. Implement the sorted list algorithm by replacing the SortedListHysteresisSimulation class.

(d) Implement the bits algorithm.

More information on how

to write your own BruteForceHysteresisSimulation, SortedListHysteresisSimulation, and BitsHysteresisSimulation classes are available on our Web site.

Acknowledgments. Portions of this work were supported under NSF DMR-9805422 and DOE DEFG02-88ER45364. An equipment grant from Intel and the support of the Cornell Theory Center are also gratefully acknowledged. We thank Harvey Gould and Jan Tobochnik for careful and helpful editing suggestions.

[1] E. Vives, J. Goicoechea, J. Ortin, and A. Planes, "Universality in models for disorder-induced phase transitions," Phys. Rev. E 52, R5 (1995), studied randombond systems of sizes up to $40 \times 40$ and mixed randomfield random-bond systems up to $100 \times 100$. Our methods are not directly applicable to random bond systems or to systems with long-range forces 2]. R. Blossey, T. Kinoshita, and J. Dupont-Roc, "Random-field Ising model for the hysteresis of the prewetting transition on a disordered substrate," Physica A 248, 247 (1998), studied the prewetting transition with our model using the efficient form of the brute force algorithm on systems up to $900 \times 900$.

[2] S. Zapperi, P. Cizeau, G. Durin, and H. E. Stanley, "Dynamics of a ferromagnetic domain wall: Avalanches, depinning transition, and the Barkhausen effect," Phys. Rev. B 58, 6353 (1998) studied the effects of long-range forces in systems with up to 32,696 spins.

[3] H. Ji and M. O. Robbins, "Percolative, self-affine, and faceted domain growth in random three-dimensional magnets," Phys. Rev. B 46, 14519 (1992), and references therein. Ji and Robbins study the closely related interface depinning model (which does not allow avalanches to start except next to existing flipped spins). The methods we discuss in this column can be used to study front propagation (by pre-flipping a layer of spins and setting the probability of flipping isolated spins to zero), but are less efficient than those developed by $\mathrm{Ji}$ and Robbins, which "forget" the spins left behind by the propagating interface. They ran $300^{3}$ and $2000^{2}$ simulations in 1991 and $12000^{2}$ simulations in 1994.

[4] J.-G. Zhu and H. N. Bertram, "Self-organized behavior in thin-film recording media," J. Appl. Phys. 69, 4709 (1991) first applied a similar model to magnets. They study a hexagonal two-dimensional model with in-plane magnetization, ferromagnetic bonds with the four neighbors along the field direction and antiferromagnetic (or missing) bonds with the two neighbors perpendicular to the field. They study systems of size $100 \times 2000$. They simulated only one value of the disorder, and found power law distributions of avalanche sizes which they attributed to self-organized criticality.

[5] J. P. Sethna, K. A. Dahmen, S. Kartha, J. A. Krumhansl, B. W. Roberts, and J. D. Shore, "Hysteresis and hierarchies: dynamics of disorder-driven first-order phase transformations," Phys. Rev. Lett. 70, 3347 (1993).

[6] In our model, the spins can flip anywhere in the region. In the fluid invasion problem, the spins can only flip next to a flipped region: the water can push into the rock only along an invading front. Our model allows the interaction of many different boundaries between up and down spin regions.

[7] See, for example, http://SimScience.org/crackling/.

[8] Neither of the useful algorithms parallelizes well; our group has made effective and extensive use of parallel machines to average over disorder. The fluctuations at the critical point are severe at all system sizes.

[9] J. P. Sethna, O. Perković, and K. A. Dahmen, in Scale Invariance and Beyond, Les Houches Workshop, March 10-14, 1997, ed. B. Dubrulle, F. Graner, and D. Sornette, Springer, Berlin, p. 87. Figure 1 (right) is incorrect in this reference: it shows $d M / d t$ for the whole hysteresis loop, not just one avalanche.

[10] O. Perković, K. A. Dahmen, and J. P. Sethna, "Avalanches, Barkhausen noise, and plain old criticality," Phys. Rev. Lett. 75, 4528 (1995); O. Perković, K. A. Dahmen, and J. P. Sethna, "Disorder-induced critical 
phenomena in hysteresis: a numerical scaling analysis," cond-mat \#9609072; O. Perković, K. A. Dahmen, and J. P. Sethna, "Disorder-induced critical phenomena in hysteresis: numerical scaling in three and higher dimensions," cond-mat \#9807336.

[11] W. H. Press, S. A. Teukolsky, and W. T. Vetterling, Numerical Recipes in C: The Art of Scientific Computing (Cambridge University Press, 1992). The book is also available online at http://www.nr.com/.

[12] NetLib is a collection of mathematical software, papers and databases at http://www.netlib.org/.

[13] The exponent $\tilde{\tau}$ can be written in terms of other critical exponents for our problem: $\tilde{\tau}=\tau+\sigma \beta \delta$.

[14] B. Drossel and K. Dahmen, "Depinning of a domain wall in the $2 \mathrm{~d}$ random-field Ising model," Eur. Phys. J. B 3, 485 (1998).

[15] A. A. Middleton, "Asymptotic uniqueness of the sliding state for charge-density waves." Phys. Rev. Lett. 68, 670 (1992); A. A. Middleton and D. S. Fisher, "Critical behavior of charge-density waves below threshold: numerical and scaling analysis," Phys. Rev. B 47, 3530 (1993).

[16] U. Wolff, "Collective Monte Carlo updating for spin systems," Phys. Rev. Lett. 62, 361 (1989).

[17] Search only those spins for which nextPossible $\left[n_{\uparrow}\right]$ has not run off the end of the list.

[18] $\left(X_{1}, X_{2}, \ldots, X_{D}\right)$ can be reduced to $X_{1}+X_{2} L+\ldots+$ $X_{D} L^{D-1}$. This reduction yields the numbering scheme shown in Figs. 5 and 6 and also allows us to store a $D$ dimensional lattice in a one-dimensional array and write code which will work in any dimension.

[19] L. Furuberg, J. Feder, A. Aharony, and T. Jøssang, "Dynamics of invasion percolation," Phys. Rev. Lett. 61, 2117 (1988).

[20] A. B. Bortz, M. H. Kalos, and J. L. Lebowitz, "A new algorithm for Monte Carlo simulation of Ising spin systems," J. Comp. Phys. 17, 10 (1975). Also see M. A. Novotny, "A New Approach to an Old Algorithm for the Simulation of Ising-like Systems," Comput. Phys. 9, 46 (1995).

[21] We use a simple algorithm to make sure that the guesses are on opposite sides of the correct guess, and then use the Brent algorithm to find the root. The Brent algorithm uses a combination of interpolation when the guesses are close, and bisection when the interpolation works badly. Descriptions and implementations of this and other root finding algorithms can be found in Refs. 11] and [12].

[22] If your random number generator is not good enough, or if you have incorrectly kept track of the number of down spins with $n_{\uparrow}$ neighbors up, your simulation may enter an infinite loop searching for the next spin. Also, if you are storing your spins in a 1D array, you should still generate $D$ random numbers, one for each coordinate. If you try to save time and only generate one random number, then for a very large system there will be gaps in the random numbers, and some spins will never be found, causing an infinite loop.

[23] One might worry that the queue will grow very large. However, the queue will only be as large as the largest shell of spins, which should scale as the surface area of the largest avalanche. Therefore, it should scale as $N^{1 / 2}$ in two dimensions, and as $N^{2 / 3}$ in three dimensions. Be- cause the avalanches tend to grow in fits and starts, this situation is even better. Note that in Fig. 1, even though the avalanche contained almost 300,000 spins, and the surface area was very large, the largest shell had only 321 spins in it (Fig. 2). The queue is generally very small compared with the system size. 